\title{
Simulating and Detecting the Quantum Spin Hall Effect in Kagomé Optical Lattice
}

\author{
Guocai Liu ${ }^{1}$, Shi-Liang Zhu ${ }^{2}$, Shaojian Jiang ${ }^{1}$, Fadi Sun ${ }^{1}$, W. M. Liu ${ }^{1}$ \\ ${ }^{1}$ Beijing National Laboratory for Condensed Matter Physics, \\ Institute of Physics, Chinese Academy of Science, Beijing 100190, China and \\ ${ }^{2}$ Laboratory of Quantum Information Technology, ICMP and SPTE, \\ South China Normal University, Guangzhou, China
}

\begin{abstract}
We propose a model which includes a nearest-neighbor intrinsic spin-orbit coupling and a trimerized Hamiltonian in the Kagomé lattice and promises to host the transition from the quantum spin Hall insulator to the normal insulator. In addition, we design an experimental scheme to simulate and detect this transition in the ultracold atom system. The lattice intrinsic spin-orbit coupling is generated via the laser-induced-gauge-field method. Furthermore, we establish the connection between the spin Chern number and the spin-atomic density which enables us to detect the quantum spin Hall insulator directly by the standard density-profile technique used in the atomic systems.
\end{abstract}

PACS numbers: 03.75.Hh, 05.30.Fk, 73.43.-f, 71.70.Ej

\section{INTRODUCTION}

Optical lattice system has gradually become a promising platform to simulate and study a lot of quantum phenomena in condensed matter physics because almost all parameters of the system can be well-controlled[1]. The recent theoretical and experimental progress in laserinduced-gauge-field [2 7] makes it a hot spot to study topological quantum states [8] in cold atoms system 9 15]. Subject to the compounds' natural properties [16], the famous Haldane model [17] proposed two decades ago has not been confirmed by experiments because the required periodic magnetic field cannot be easily implemented in actual material. For topological insulator, an important extension from the Haldane model to a timereversal invariant system [18 23], only a few materials are confirmed currently to have such exotic topological properties in the nature [24 27], because the existence of such properties require relatively strong spin-orbit (SO) coupling. However, in optical lattice system, we can engineer the lattice Hamiltonian to guarantee that this system hosts these novel topological phases [14]. Up to now, the neutral-cold-atom integer and fractional quantum Hall effects have been studied [28 30] and also the realization of Haldane model has been designed by using the laserinduced-gauge-field method in optical lattice [12].

In this paper, we propose a scheme to simulate and detect the 2-dimensional (2D) quantum spin Hall (QSH) insulator in a Kagomé optical lattice with a trimer and a nearest-neighbor SO coupling term. With laser-inducedgauge-field method, one can design a variety of lattice SO couplings [5, 31 33], which is convenient for us to study the $2 \mathrm{D}$ topological insulator in the optical lattice [9-11]. However, the original proposal [18] of realizing 2D QSH insulator in honeycomb lattice requires the next-nearestneighbor hopping amplitude. This requirement causes doing experiments in the optical lattice difficult because high barrier makes the next-nearest-neighbor tunneling very small. Recently, a model [34] raised in Kagomé lattice also requires the next-nearest-neighbor hopping. Interestingly, there is another QSH insulator model in the complicated Kagomé lattice. The inspiration comes from the fact that spin chirality in ferromagnetic Kagomé lattice exerts important effects on orbital magnetic moment and anomaly quantum Hall effect [35]. We find that a Kagomé optical lattice with the trimer and SO coupling terms can host the 2D QSH insulator phase with only the nearest-neighbor hopping. Since the model only involves the nearest-neighbor hopping, it would be easier to be implemented in cold atomic experiments.

Furthermore, compared to the condensed matter systems, we find that detecting QSH insulator has more advantage in optical lattice system. Due to the timereversal symmetry, Chern number can not be taken as a topological invariant to characterize the QSH insulator in real electron system [36]. Chern number $C_{\uparrow}=+1$ $\left(C_{\downarrow}=-1\right)$ in a QSH phase for the up-spin (down-spin) electrons and the total Chern number $C=C_{\uparrow}+C_{\downarrow}=0$. Since one can not distinguish the contributions of the conductance from the up or down spin electrons at current technology, it is impossible to determine whether the system is in the QSH phase or normal phase by measuring Hall conductance. However, in cold atomic systems, it is the atom's internal states that represent the spin, not real spin, which brings certain benefits to measurement. One can directly measure the spin Chern number to determine whether the system lies in the QSH phase or not because optically measuring the atomic internal states is very simple. In this paper, we demonstrate that the method developed to detect the Chern number in cold atomic systems [12, 30] can be put forward further to measure the spin Chern number, and thus we establish the connection between the spin Chern number and the spin-atomic density which enables us to detect the topological Chern numbers directly by the standard densityprofile technique used in the atomic systems.

The paper is organized as follows: In Sec. II, we introduce the model in Kagomé lattice with both trimer and spin-orbital coupling. This model can realize the QSH phase with only the nearest-neighbor hoping terms; in Sec. III, we explain how to simulate this model in coldatom optical lattice, which includes designing the lat- 
(a)

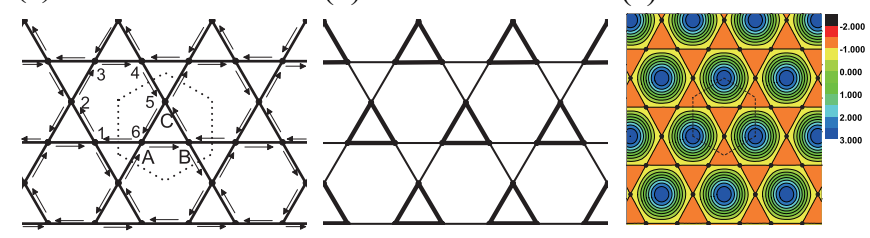

FIG. 1. (Color online) (a) Schematic picture of the nearest neighbor intrinsic SO coupling in 2D Kagomé lattice. The up-spin atoms hop along (against) the arrowed direction with amplitude $i \lambda_{\mathrm{SO}}\left(-i \lambda_{\mathrm{SO}}\right)$. For the down-spin atoms, the arrows are reversed. The dashed line represents the WignerSeitz unit cell, which contains three independent sites (A, B, $\mathrm{C})$. (b) the trimer Kagomé lattice. Hopping amplitude corresponds to $t+\kappa(t-\kappa)$ for the thick (thin) bonds. (c) Contours of the effective magnetic field for up-spin atoms defined by Eq. (21).

tice SO coupling by using the laser-induced-gauge-field method; we present the method to detect the QSH phase in Sec. IV and give a brief summary in Sec. V.

\section{MODEL}

Let us consider the tight-binding model for twocomponent fermionic atoms on the Kagomé optical lattice, which consists of three triangular sublattices A, B and $\mathrm{C}$ (Fig. 1). The spin-independent part of the Hamiltonian is given by

$$
\begin{aligned}
H_{0}= & t_{0} \sum_{m n \alpha}\left(b_{m, n, \alpha}^{\dagger} a_{m, n, \alpha}+b_{m-1, n, \alpha}^{\dagger} a_{m, n, \alpha}\right. \\
& +c_{m, n, \alpha}^{\dagger} b_{m, n, \alpha}+c_{m+1, n-1, \alpha}^{\dagger} b_{m, n, \alpha} \\
& \left.+a_{m, n, \alpha}^{\dagger} c_{m, n, \alpha}+a_{m, n+1, \alpha}^{\dagger} c_{m, n, \alpha}\right)+ \text { H.c. },
\end{aligned}
$$

where $t_{0}$ is the hopping amplitude between the nearest neighbor link, $(m, n)$ labels the Kagomé unit cells with the unit vectors $\mathbf{b}_{1}=(2,0) a$ and $\mathbf{b}_{2}=(1, \sqrt{3}) a, a_{m, n, \alpha}^{\dagger}$ $\left(a_{m, n, \alpha}\right)$ is the creation (annihilation) operator of an atom with spin $\alpha$ (up or down) on lattice site $(m, n)$ on sublattice A (an equivalent definition is used for sublattice $\mathrm{B}$ and $\mathrm{C}$ ). For simplicity, we choose $t_{0}=1$ as the energy unit and the distance between the nearest sites $a$ as the length unit throughout this paper.

By using the Fourier transform of atomic operators $a_{m, n, \alpha}$, i.e.,

$$
a_{m, n, \alpha}=\frac{1}{\sqrt{N}} \sum_{\mathbf{k}} a_{\mathbf{k} \alpha} e^{-i \mathbf{k} \cdot \mathbf{R}_{m n}^{A}}
$$

the Hamiltonian (10) can be diagonalized in the momentum space as

$$
H_{0}=\sum_{\mathbf{k}} \psi_{\mathbf{k}}^{+}\left(\mathcal{H}_{0}(\mathbf{k}) \otimes \mathbf{I}_{2 \times 2}\right) \psi_{\mathbf{k}}
$$

where $\mathbf{I}_{2 \times 2}$ is a $2 \times 2$ unit matrix. The six-component basis operator $\psi_{\mathbf{k}}=\left(a_{\mathbf{k} \uparrow}, b_{\mathbf{k} \uparrow}, c_{\mathbf{k} \uparrow}, a_{\mathbf{k} \downarrow}, b_{\mathbf{k} \downarrow}, c_{\mathbf{k} \downarrow}\right)^{\mathrm{T}}$ describes an atom in the corners of the Kagomé lattice unit cell (Fig. 1) with spin $\alpha(\uparrow$ and $\downarrow)$. Here $\mathcal{H}_{0}(\mathbf{k})$ is a $3 \times 3$ spinless matrix given by

$$
\mathcal{H}_{0}(\mathbf{k})=\left(\begin{array}{ccc}
0 & 2 \cos P_{1} & 2 \cos P_{3} \\
2 \cos P_{1} & 0 & 2 \cos P_{2} \\
2 \cos P_{3} & 2 \cos P_{2} & 0
\end{array}\right)
$$

where we have defined $P_{1}=\mathbf{k} \cdot \mathbf{a}_{1}, P_{2}=\mathbf{k} \cdot \mathbf{a}_{2}, P_{3}=\mathbf{k} \cdot \mathbf{a}_{3}$ with $\mathbf{a}_{1}=(1,0), \quad \mathbf{a}_{2}=(-1 / 2, \sqrt{3} / 2), \quad$ and $\mathbf{a}_{3}=(-1 / 2,-\sqrt{3} / 2)$ representing the displacements in a unit cell from $\mathrm{A}$ to $\mathrm{B}$ site, from $\mathrm{B}$ to $\mathrm{C}$ site, and from $\mathrm{C}$ to $\mathrm{A}$ site, respectively. In this notation, the first Brillouin zone is a hexagon with the corners of $\mathbf{K}= \pm(2 \pi / 3) \mathbf{a}_{1}, \pm(2 \pi / 3) \mathbf{a}_{2}, \pm(2 \pi / 3) \mathbf{a}_{3}$, and two of which are independent.

The energy spectrum for spinless Hamiltonian $\mathcal{H}_{0}(\mathbf{k})$ is characterized by one dispersionless flat band $\left(\epsilon_{1 \mathbf{k}}^{(0)}=-2\right)$, which reflects the fact that the $2 \mathrm{D}$ Kagomé lattice is a line graph of the honeycomb structure [37], and two dispersive bands, $\epsilon_{2(3) \mathbf{k}}^{(0)}=1 \mp \sqrt{4 b_{\mathbf{k}}-3}$ with $b_{\mathbf{k}}=\sum_{i=1}^{3} \cos ^{2}\left(\mathbf{k} \cdot \mathbf{a}_{i}\right)$. These two dispersive bands touch at Dirac points $\mathbf{K}$ and exhibit a cusp, $\epsilon_{2(3) \mathbf{k}}^{(0)}=(1 \mp \sqrt{3}|\mathbf{k}-\mathbf{K}|)$.

When the following intrinsic SO coupling term is taken into account in the Kagomé lattice model, as illustrated in Fig. 1(a), the gap will be opened at the two inequivalent Dirac points. The tight-binding expression for this SO coupling Hamiltonian can be given as follows:

$$
\begin{aligned}
H_{\mathrm{SO}} & =i \lambda_{\mathrm{SO}} \sum_{m, n}\left(b_{m, n}^{\dagger} \sigma_{z} a_{m, n}+b_{m-1, n}^{\dagger} \sigma_{z} a_{m, n}\right. \\
& +c_{m, n}^{\dagger} \sigma_{z} b_{m, n}+c_{m+1, n-1}^{\dagger} \sigma_{z} b_{m, n} \\
& \left.+a_{m, n}^{\dagger} \sigma_{z} c_{m, n}+a_{m, n+1}^{\dagger} \sigma_{z} c_{m, n}\right)+ \text { H.c. },
\end{aligned}
$$

where $\lambda_{\mathrm{SO}}$ is the SO coupling constant, $\sigma_{z}$ is the Pauli matrix and $a_{m, n}^{\dagger}=\left(a_{m, n, \uparrow}^{\dagger}, a_{m, n, \downarrow}^{\dagger}\right)$. Taking the Fourier transform (2) and considering the $\psi_{\mathbf{k}}$ below Eq. (3), we have $H_{\mathrm{SO}}=\sum_{\mathbf{k}} \psi_{\mathbf{k}}^{+} \mathcal{H}_{\mathrm{SO}}(\mathbf{k}) \psi_{\mathbf{k}}$, where

$$
\mathcal{H}_{\mathrm{SO}}(\mathbf{k})=\left(\begin{array}{cc}
\mathcal{H}_{+}(\mathbf{k}) & 0 \\
0 & \mathcal{H}_{-}(\mathbf{k})
\end{array}\right)
$$

with

$$
\mathcal{H}_{ \pm}(\mathbf{k})= \pm 2 i \lambda_{\mathrm{SO}}\left(\begin{array}{ccc}
0 & -\cos P_{1} & \cos P_{3} \\
\cos P_{1} & 0 & -\cos P_{2} \\
-\cos P_{3} & \cos P_{2} & 0
\end{array}\right)
$$

This SO coupling destroys spin SU(2) symmetry and opens a band gap $\Delta_{\mathrm{SO}}=\sqrt{3} \lambda_{\mathrm{SO}}$ at Dirac point.

Lattice trimerization can break inversion symmetry of Kagomé lattice and also open a gap at Dirac point [34]. 
It is described by

$$
\begin{aligned}
H_{\text {trim }}= & \sum_{m n \alpha}\left[\kappa\left(b_{m, n, \alpha}^{\dagger} a_{m, n, \alpha}-b_{m-1, n, \alpha}^{\dagger} a_{m, n, \alpha}\right)\right. \\
& +\kappa\left(c_{m, n, \alpha}^{\dagger} b_{m, n, \alpha}-c_{m+1, n-1, \alpha}^{\dagger} b_{m, n, \alpha}\right) \\
& \left.+\kappa\left(a_{m, n, \alpha}^{\dagger} c_{m, n, \alpha}-a_{m, n+1, \alpha}^{\dagger} c_{m, n, \alpha}\right)\right]+ \text { H.c }
\end{aligned}
$$

where $\kappa$ describe an alternating pattern of bond hopping integrals along the three principal spatial directions as illustrated in Fig. 1(b). Taking the Fourier transform (2) again, the trimerized Hamiltonian can be rewritten as $H_{\text {trim }}=\sum_{\mathbf{k}} \psi_{\mathbf{k}}^{+}\left(\mathcal{H}_{\text {trim }}(\mathbf{k}) \otimes \mathbf{I}_{2 \times 2}\right) \psi_{\mathbf{k}}$ with

$$
\mathcal{H}_{\text {trim }}(\mathbf{k})=2 i\left(\begin{array}{ccc}
0 & -\kappa \sin P_{1} & \kappa \sin P_{3} \\
\kappa \sin P_{1} & 0 & -\kappa \sin P_{2} \\
-\kappa \sin P_{3} & \kappa \sin P_{2} & 0
\end{array}\right)
$$

for both spin components.

We take above SO coupling and trimerized Hamiltonian as perturbation, which means $\lambda_{\mathrm{SO}} \ll t$ and $\kappa \ll t$. Although both of perturbations can bring gaps at Dirac points independently, these two gaps have different topological nature. As we will show in the following, the former is non-trivial and quantum spin Hall effect will occur if Fermi energy level locates in the gap; the latter is a trivial gap.

In order to prove above assertion, we expand the total Hamiltonian $\mathcal{H}(\mathbf{k})=\mathcal{H}_{0}(\mathbf{k})+\mathcal{H}_{\mathrm{SO}}(\mathbf{k})+\mathcal{H}_{\text {trim }}(\mathbf{k})$ at two inequivalent points $\mathbf{K}_{ \pm}=\left( \pm \frac{2 \pi}{3}, 0\right)$, then take $\mathbf{k} \cdot \mathbf{p}$ perturbation theory to get its effective Hamiltonian. At last, projecting it onto bands 2 and 3 subspace, we get four independent Dirac Hamiltonian,

$$
\mathcal{H}_{s \sigma}^{K}=-s v_{F} k_{x} \tau_{z}+s v_{F} k_{y} \tau_{x}+m_{s \sigma} \tau_{y}
$$

where $s= \pm 1$ and $\sigma= \pm 1$ represent different valleys $\mathbf{K}_{ \pm}$ and spin indices, respectively, $v_{F}=\sqrt{3} t$ is Fermi velocity, and $\tau_{i}$ are Pauli matrices with $i=x, y, z$. The Dirac mass $m_{s \sigma}=\sqrt{3} \sigma \lambda_{\mathrm{SO}}-3 s \kappa$.

We now turn to address the topological properties of this model. The system is in the QSH phase when the SO coupling dominates in the condition $\left|\lambda_{\mathrm{SO}}\right|>|\sqrt{3} \kappa|$; otherwise, the system is in the normal phase. It can be proved by directly calculating the $Z_{2}$ topological invariant [18, 20]. Alternatively, one can also understand the topological phases from the view of spin Chern number [36]. In order to do that, we apply a uniform magnetic field along $z$-direction with gauge vector $\mathbf{A}(\mathbf{r})=$ $(0, \mathcal{B} x, 0)$ and obtain the Landau energy levels

$$
E_{n}^{s \sigma}= \begin{cases}m_{s \sigma} \operatorname{sgn}(e \mathcal{B}) & n=0 \\ \pm \sqrt{2 n \hbar v_{F}^{2}|e \mathcal{B}|+m_{s \sigma}^{2}} & n=1,2,3, \cdots\end{cases}
$$

By using the Green function theory [12], we can get the Chern number

$$
C_{s \sigma}=\frac{1}{2} \operatorname{sgn}\left(E_{0}^{s \sigma}\right)=\frac{1}{2} \operatorname{sgn}\left(m_{s \sigma} \operatorname{sgn}(e \mathcal{B})\right)
$$

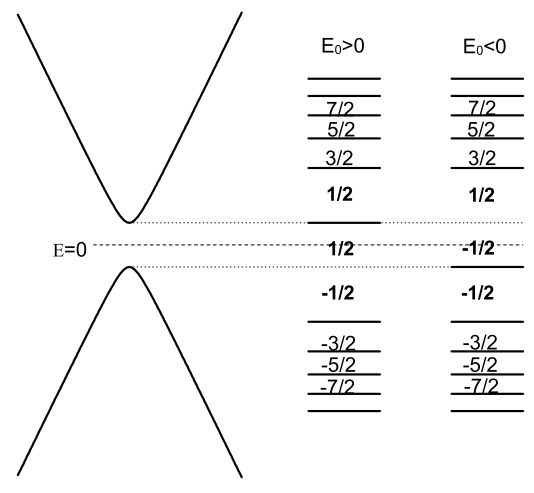

FIG. 2. (Color online) Illustration of the effect on Chern number by the sign of $n=0$ Landau energy level. The left figure corresponds to disperse relation of Eq. (10) without magnetic field. The right two figure show the Chern numbers when magnetic field is applied and Fermi energy locate at different interval for two cases with $E_{0}>0$ and $E_{0}<0$. Here $E_{0}$ denotes the $n=0$ Landau energy level. The dashed line represents the zero energy and the doted lines give the original band gap.

between the energy interval $-\left|m_{s \sigma}\right|<\mu<\left|m_{s \sigma}\right|$ with $\mu$ being the Fermi energy (Fig. 2). Actually it is the sign of $n=0$ energy (zero-mode) which determines the Chern number. As an example, We focus on $\mu=0$ and take $e \mathcal{B}>0$ throughout this paper to see the difference more clearly between QSH phase and normal phase. For QSH phase, we take $\lambda_{\mathrm{SO}}>\sqrt{3} \kappa>0$, and it is easy to see that, for up-spin atoms, $m_{s= \pm 1, \uparrow}>0$ and then $\mathrm{C}_{\uparrow}=\mathrm{C}_{+1, \uparrow}+C_{-1, \uparrow}=1$. For down-spin atom, $m_{s= \pm 1, \downarrow}<0$ and $\mathrm{C}_{\downarrow}=\mathrm{C}_{+1, \downarrow}+C_{-1, \downarrow}=-1$. However, if we take $\sqrt{3} \kappa>\lambda_{\mathrm{SO}}>0$, which corresponds to the normal phase, $\mathrm{C}_{\uparrow}=\mathrm{C}_{\downarrow}=0$ for both up- and down-spin atoms. In the QSH phase, the Chern numbers with different spin components have same value but with opposite sign. Whereas if it is in normal phase, the Chern numbers equal to zero for both up- and down-spin atoms.

To further understanding the topological properties of the model, we show the edge state effects in Fig. 3. From Fig. 3(a), we can see that there is a pair of chiral gapless edge states for every band gap when the SO coupling dominates. This means that the system is in topological insulator phases at $1 / 3$ - and $2 / 3$-filling. When only trimer term exists, it opens a band gap at Dirac point but no edge states connect the upper and lower bands (Seeing Fig. 3(b)), therefore the system is in normal insulator phase at $2 / 3$-filling. On the other hand we also see that the trimer term cannot open a gap between the band 1 and 2 (Fig. 3(b)). Therefore the system at $1 / 3$-filling will be still in the topological insulator phase when two perturbations are present but the trimer term dominates (Seeing Fig. 3(c)). 

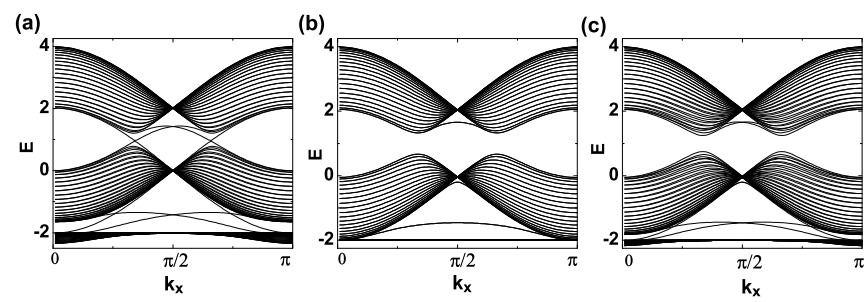

FIG. 3. (Color online) The band structure of the lattice model in the stripe geometry. We take $\lambda_{\mathrm{SO}}=0.1, \kappa=0$ for $(\mathrm{a}), \lambda_{\mathrm{SO}}=0$, $\kappa=0.1$ for (b) and $\lambda_{\mathrm{SO}}=0.05, \kappa=0.1$ for (c).

\section{SIMULATION}

In this section we introduce an approach to simulate the Kagomé lattice with the trimer and SO coupling terms in an optical lattice system. To this end, two problems need to be solved. One is how to generate the Kagomé optical lattice with the trimer terms; the other is how to simulate the lattice SO coupling. As for the first problem, we use the superlattice technique addressed in Refs. [38 40], that is, three super-laser beams with the same wave vector length but different polarizations are applied along three different directions: $\mathbf{e}_{y}$ and $\frac{1}{2} \mathbf{e}_{y} \pm \frac{\sqrt{3}}{2} \mathbf{e}_{x}$, respectively. In our proposal, each superlaser beam consists of four large detuned standing-wave lasers with the same polarization but different wave vector length in the $x-y$ plane. The total potential is thus given by

$$
\begin{aligned}
V(\mathbf{r})= & V_{0} \sum_{i=1}^{3}\left[\cos \left(\mathbf{k}_{i} \cdot \mathbf{r}+3 \delta_{i} \varphi / 2\right)+2 \cos \left(\mathbf{k}_{i} \cdot \mathbf{r} / 3+\delta_{i} \varphi / 2\right)\right. \\
& +4 \cos \left(\mathbf{k}_{i} \cdot \mathbf{r} / 9+\delta_{i} \varphi / 6\right) \\
& \left.+\zeta \cos \left(\mathbf{k}_{i} \cdot \mathbf{r} / 9+\delta_{i}(\varphi / 6+\pi / 2)\right)\right]^{2}
\end{aligned}
$$

with the wave vectors $\mathbf{k}_{1}=\left(\frac{\sqrt{3}}{2}, \frac{1}{2}\right) k, \mathbf{k}_{2}=\left(-\frac{\sqrt{3}}{2}, \frac{1}{2}\right) k$, $\mathbf{k}_{3}=(0,1) k$ and $\delta_{1}=\delta_{2}=-\delta_{3}=1$. Firstly, we consider the case with $\zeta=0$. One can get a triangular lattice when $\varphi=0$ or $2 \pi$ and a Kagomé lattice when $0<\varphi<2 \pi$. A uniform Kagomé lattice corresponds to $\varphi=\pi$, as shown in Fig. 4(a). When $\varphi$ takes other values, one can obtain the trimerized Kagomé lattice accompanying distortion of the lattice structure [38-40]. When we increase the strength of trimerized Hamiltonian, the Kagomé lattice will be distorted. To overcome this defect, we add another laser beam which corresponds to the $\zeta \neq 0$ in the Eq. (13) and assume $\varphi=\pi$ all the time. The added laser will interfere with primary lasers and generate the trimerized Kagomé lattice. $\zeta$ is an adjustable parameter to control the strength of trimerized Hamiltonian. With this method, the Kagome lattice will not have obvious offset from the uniform one even if $\zeta$ takes a relative large value. As shown in Fig. 4(b), the parameter $\zeta=1.5$ is chosen as a typical example.

We now focus on how to simulate the lattice SO cou- pling. Using the laser-induced-gauge-field method, it was proposed that both Abelian and non-Abelian gauge fields can be simulated in cold atomic system. In addition, the experiments to achieve such artificial gauge fields have been reported [6, 7]. Interestingly, it was proposed that a periodic magnetic field, which is not easy to be realized in a condensed-matter system, can be created by two opposite-traveling standing-wave laser beams [5]. In the following, we will show in an explicit manner that the artificial gauge field proposed in Ref. [5] is equivalent to a SO coupling. Furthermore, the required lattice SO coupling addressed in the previous section can also be achieved in a suitable configuration of the laser beams.

In optical lattice systems, cold atoms can hop between adjacent sites. According to Peierls theory, the additional gauge vector potential $\mathbf{A}$ makes the hopping obtain a phase factor $\exp \left(i \frac{e}{\hbar} \int \mathbf{A} \cdot \mathbf{d l}\right)$, where the integral along the hopping path. If the atom has multiple states, which correspond to different spin components, vector potential $\mathbf{A}$ should be a matrix. After taking such an approximation, that is $t_{i j}^{\alpha \beta}=t_{i j}$, here $\alpha, \beta$ on behalf of any spin index, the correction to atom hopping between different sites coming from gauge field is equivalent to a unitary operator [4]. For a two-component atom system, the unitary operator can be written as

$$
U_{i j}=e^{i \alpha_{i j} \sigma_{i j}}=\cos \alpha_{i j}+i \sigma_{i j} \sin \alpha_{i j},
$$

where $i, j$ represent different site indices, $\alpha_{i j}$ is the gauge flux and depends on the hopping integral. Here $\sigma_{i j}$ is the Pauli matrix, whose specific form depends on the gauge vector potential. Therefore, a tight-binding Hamiltonian of atoms can be written as

$$
\bar{H}=\sum_{\langle i, j\rangle}\left(t_{i j} \bar{a}_{j}^{\dagger} U_{i j} \bar{a}_{i}+\text { H.c. }\right)=\bar{H}_{0}+\bar{H}_{\mathrm{SO}}
$$

with

$$
\begin{aligned}
\bar{H}_{0} & =\sum_{\langle i, j\rangle}\left(t_{i j} \cos \alpha_{i j}\right) \bar{a}_{j}^{\dagger} \bar{a}_{i}+\text { H.c. } \\
\bar{H}_{\mathrm{SO}} & =i \sum_{\langle i, j\rangle}\left(t_{i j} \sin \alpha_{i j}\right) \bar{a}_{j}^{\dagger} \sigma_{i j} \bar{a}_{i}+\text { H.c.. }
\end{aligned}
$$

Here $\langle i, j\rangle$ denotes the nearest-neighbor hoping, $\bar{a}_{i}\left(\bar{a}_{i}^{\dagger}\right)$ the creation (annihilation) operator on site $i$. The first term is the normal Hamiltonian, while the second one is equivalent to $\mathrm{SO}$ coupling. From above equations, we can observe that: (i) By choosing suitable Pauli matrix $\sigma_{i j}$, one can simulate various $\mathrm{SO}$ coupling existing in actual materials. For our model, we should choose $\sigma_{i j}=\sigma_{z}$. (ii) Through adjusting the gauge flux $\alpha_{i j}$, one can change relative strength between the two terms. For example, $\alpha_{i j}=0$ corresponds no SO coupling, while $\alpha_{i j}=\pi / 2$ is equivalent to only the SO coupling interaction existed in the system. This facilitates us to study the nature brought by SO coupling.

We now turn to address the approach to achieve the intrinsic SO coupling described in Eq.(5). To this end, we 


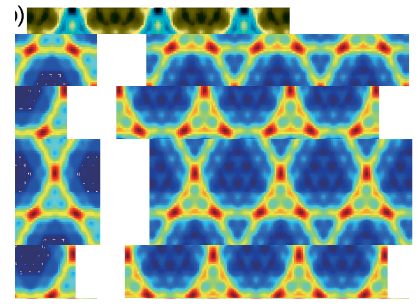

FIG. 4. (Color online) (a) A uniform Kagomé lattice for $\varphi=\pi$ and $\zeta=0$. (b) A trimer Kagomé lattice for $\varphi=\pi$ and $\zeta=1.5$.

consider a cold atomic system with each atom having an $\Lambda$-type level configuration (Fig. 5(a)). The ground states $|1\rangle$ and $|2\rangle$ are coupled to the excited state $|3\rangle$ through spatially varying standing-wave laser field, with Rabi frequencies $\Omega_{p}=\Omega \sin \theta e^{-i S_{1}}$ and $\Omega_{c}=\Omega \cos \theta e^{-i S_{2}}$, respectively. With rotating-wave approximation, the laseratom coupling Hamiltonian is given by

$$
\hat{H}_{\mathrm{int}}=-\frac{\hbar}{2}\left(\begin{array}{ccc}
0 & 0 & \Omega_{p} \\
0 & 0 & \Omega_{c} \\
\Omega_{p}^{*} & \Omega_{c}^{*} & -2 \Delta
\end{array}\right)
$$

with the eigenstates (the dressing states)

$$
\begin{aligned}
& \left|\chi_{1}\right\rangle=e^{-i S_{1}} \cos \theta|1\rangle-e^{-i S_{2}} \sin \theta|2\rangle \\
& \left|\chi_{2}\right\rangle=\cos \varphi \sin \theta e^{-i S_{1}}|1\rangle+\cos \varphi \cos \theta e^{-i S_{2}}|2\rangle-\sin \varphi|3\rangle \\
& \left|\chi_{3}\right\rangle=\sin \varphi \sin \theta e^{-i S_{1}}|1\rangle+\sin \varphi \cos \theta e^{-i S_{2}}|2\rangle+\cos \varphi|3\rangle
\end{aligned}
$$

and eigenvalues $\lambda_{n=1,2,3}=0, \frac{\hbar}{2}\left(\Delta \mp \sqrt{\Delta^{2}+\Omega^{2}}\right)$. Here, single-photon detuning $\Delta=\omega_{3}-\omega_{1}-\omega_{p}$, with $\omega_{3}$, $\omega_{1}, \omega_{p}$ the intrinsic frequency of atom states $|3\rangle$, $|1\rangle$ and laser $\Omega_{p}$, respectively. In the new basis space $|\chi\rangle=\left\{\left|\chi_{1}\right\rangle,\left|\chi_{2}\right\rangle,\left|\chi_{3}\right\rangle\right\}$, the primary atom Hamiltonian $\hat{H}=\frac{\mathbf{p}^{2}}{2 M}+\hat{H}_{\text {int }}(\mathbf{r})+\hat{V}(\mathbf{r}) \quad$ can be rewritten as $H=\frac{1}{2 M}(-i \hbar \nabla-\mathbf{A})^{2}+V$ with $M$ the atom mass, $\mathbf{A}$ and $V$ being matrix with matrix element $\mathbf{A}_{n, m}=i \hbar\left\langle\chi_{n}(\mathbf{r}) \mid \nabla \chi_{m}(\mathbf{r})\right\rangle$, $V_{n, m}=\lambda_{n}(\mathbf{r}) \delta_{n, m}+\left\langle\chi_{n}(\mathbf{r})|\hat{V}(\mathbf{r})| \chi_{m}(\mathbf{r})\right\rangle, \quad$ respectively. One can see that in the new basis the atom can be considered as moving in gauge potential $\mathbf{A}$, which corresponds to an effective magnetic field $\mathbf{B}_{\text {eff }}=(\nabla \times \mathbf{A})-\frac{i}{\hbar}(\mathbf{A} \times \mathbf{A})$ [2, 12].

We focus on the subspace spanned by the two lower eigenstates $\left\{\left|\chi_{1}\right\rangle,\left|\chi_{2}\right\rangle\right\}$, which is redefined by $\left|\chi_{\uparrow}\right\rangle \equiv\left|\chi_{1}\right\rangle$ and $\left|\chi_{\downarrow}\right\rangle \equiv\left|\chi_{2}\right\rangle$ with the spin language. This gives an effective spin- $1 / 2$ system. For the large detuning $(\Delta \gg \Omega)$ case, both states $\left|\chi_{\uparrow}\right\rangle$ and $\left|\chi_{\downarrow}\right\rangle$ are stable under atomic spontaneous emission from the initial excite state $|3\rangle$. Furthermore, we assume the adiabatic condition, which requires the off-diagonal elements of the matrices (a)

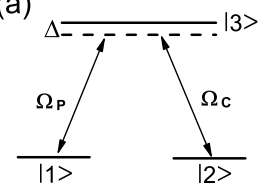

(b)

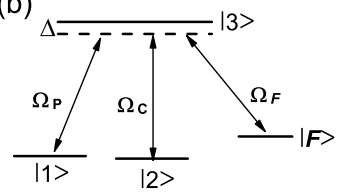

FIG. 5. (Color online) (a) Illustration of the three-level $\Lambda$ type atoms coupling with two laser beams with Rabi frequencies $\Omega_{p}$ and $\Omega_{c}$. (b) Another different hyperfine level $|F\rangle$ for detection which can be coupled to the excited state $|3\rangle$ by Rabi frequency $\Omega_{F}$.

A are smaller than the eigenenergy differences $\left|\lambda_{m}-\lambda_{n}\right|$ $(m, n=1,2,3)$ of the states $\left|\chi_{m}\right\rangle$. Under this adiabatic condition and in the basis space $\left\{\left|\chi_{\uparrow}\right\rangle,\left|\chi_{\downarrow}\right\rangle\right\}$, the gauge potential A becomes diagonal and takes the form [5]

$$
\mathbf{A}=\left(\begin{array}{cc}
\mathbf{A}_{\uparrow} & 0 \\
0 & \mathbf{A}_{\downarrow}
\end{array}\right)
$$

with

$$
\mathbf{A}_{\uparrow}=-\mathbf{A}_{\downarrow}=\hbar\left(\nabla S_{1} \cos ^{2} \theta+\nabla S_{2} \sin ^{2} \theta\right) .
$$

Here we neglect the correction to nearest-neighbor tunnelling brought by the change of potential $V$ (r) because of the large detuning approximation.

We consider a specific configuration of the laser beams with two opposite-travelling standingwave laser beams 5, 12, which take the Rabi frequencies $\quad \Omega_{p}=\Omega \sin \left(k_{2} y+\frac{\pi}{4}\right) e^{i\left(k_{1} x+k_{z} z\right)}$ and $\quad \Omega_{c}=\Omega \cos \left(k_{2} y+\frac{\pi}{4}\right) e^{-i\left(k_{1} x+k_{z} z\right)}$. The effective gauge potential is generated as $\mathbf{A}_{\uparrow}=-\mathbf{A}_{\downarrow}=\hbar \sin \left(2 k_{2} y+2 \phi_{1}\right)\left(k_{1} \mathbf{e}_{x}+k_{z} \mathbf{e}_{z}\right) . \quad$ Here $k_{1}=k \sin \theta_{1} \cos \theta_{2}, \quad k_{2}=k \cos \theta_{1}, \quad$ and $\quad k_{z}=k \sin \theta_{1} \sin \theta_{2}$ with $k$ the wave vector number of laser, $\theta_{1}$ the angle between the wave vector and $\mathbf{e}_{y}$ axis, $\theta_{2}$ the angle between $\mathbf{e}_{x}$ axis and the plane consisting of wave vector and $\mathbf{e}_{y}$ axis. We emphasize that the choice of wave vector $k_{2}$ of the laser beams must be a multiple of $\pi / \sqrt{3}$ in order to be commensurate with the optical lattice. We take $k_{2}=\pi / \sqrt{3}$. The Peierls phase factors for the nearest neighbor hopping in Fig. 1(a) are $\varphi_{12}^{\alpha}=\varphi_{23}^{\alpha}=\varphi_{45}^{\alpha}=\varphi_{56}^{\alpha}=-\alpha \frac{\sqrt{3} k_{1}}{3 k_{2}}=-\alpha \frac{k_{1}}{\pi} \quad$ and $\quad \varphi_{34}^{\alpha}=\varphi_{61}^{\alpha}=0$ with $\alpha= \pm 1$ representing the up- and down-spin. Considering the symmetry of Kagomé lattice, the vector potential $\mathbf{A}$ is rotated by $\pm 2 \pi / 3$ to obtain the other two vector potentials. Therefore, the total effective vector potential and magnetic field can be written as

$$
\begin{aligned}
\mathbf{A}_{e f f}^{\alpha}= & \alpha \hbar k_{1}\left[\left(\sin \left(2 k_{2} y\right)-\cos \left(k_{2} y\right) \sin \left(\sqrt{3} k_{2} x\right)\right) \mathbf{e}_{x}\right. \\
& \left.+\sqrt{3} \sin \left(k_{2} y\right) \cos \left(\sqrt{3} k_{2} x\right) \mathbf{e}_{y}\right], \\
\mathbf{B}_{e f f}^{\alpha}= & -\alpha \frac{2 \pi \hbar k_{1}}{\sqrt{3}}\left[2 \sin \left(k_{2} y\right) \sin (\pi x)+\cos \left(2 k_{2} y\right)\right] \mathbf{e}_{z} .
\end{aligned}
$$


It should be noticed that we have dropped the $\mathbf{e}_{z}$ component in Eq. (20) because the integral for Peierls phase is only in $x-y$ plane. The contours of the magnetic field for up-spin are plotted in Fig. 1(c). However, the total accumulated phases for the nearest-neighbor hopping along the arrowed directions in Fig. 1(a) are

$$
\varphi_{61}^{\alpha}=\varphi_{45}^{\alpha}=\varphi_{34}^{\alpha}=\varphi_{12}^{\alpha}=\varphi_{23}^{\alpha}=\varphi_{56}^{\alpha}=-\alpha \frac{2 k \sin \theta_{1} \cos \theta_{2}}{\pi}=\alpha \varphi .
$$

We must retain $\theta_{1}$ to satisfy $k_{2}=\pi / \sqrt{3}$. However, we can alter $\varphi$, which controls the relative strength between SO coupling and ordinary hopping terms, by changing the angle $\theta_{2}$ in the $x-z$ plane. So, we can replace $t_{i j} \cos \alpha_{i j} \rightarrow t \cos \varphi$ in Eq. (16) and $t_{i j} \sin \alpha_{i j} \rightarrow t \sin \varphi \rightarrow \lambda_{\mathrm{SO}}$ in Eq. (17) and therefore get the intrinsic SO couping model in our cold-atomic Kagomé optical lattice.

\section{DETECTION}

For quantum Hall effect in two-dimensional electronic gas in condensed matter system, Hall conductivity $\sigma_{x y}$ and Chern number satisfy the relation $\sigma_{x y}=\frac{e^{2}}{h} C$ with $h$ the Planck constant and $e$ electronic charge. Thus one can usually detect the Chern number through measuring Hall conductivity. However, the detection of the spin Chern number in actual material system is challenge because one can not distinguish the contributions of the conductivity from up- and down-spin electrons, respectively.

Unlike in electronic system, we will show that a significant advantage of atomic system is that the spin Chern number can be directly verified by using the similar method to detect the (mass) Chern number. It has been shown that the conductivity $\sigma_{x y}$ (Chern number) is related to the atomic density from the Streda formula $\sigma_{x y}=\partial \rho /\left.\partial \mathcal{B}\right|_{\mu, T}$ when a uniform magnetic field $\mathcal{B}$ is applied in the system. Thus one can measure the Chern number through the detection of the density profile, which is a standard detection method used in atomic system [12, 30]. Since the internal state-dependent image has also been achieved, the similar method can be straightforward expanded to measure the spin Chern number. Therefore we may establish the relation between the spin Chern and atom density, enabling us to detect the OSH phase of the system via density-profilemeasurement technique.

We now introduce how to detect the QSH phase in ultracold-atomic optical lattice. In cold atomic system, the informations of quantum states are usually measured from the density profile image. So we will first derive spin-atomic density from the Dirac Hamiltonian (10), and then obtain the information of the Chern number of the system.

The uniform magnetic field can be simulated by rotating the optical lattice at a constant frequency $\omega=e \mathcal{B} / 2 M$.
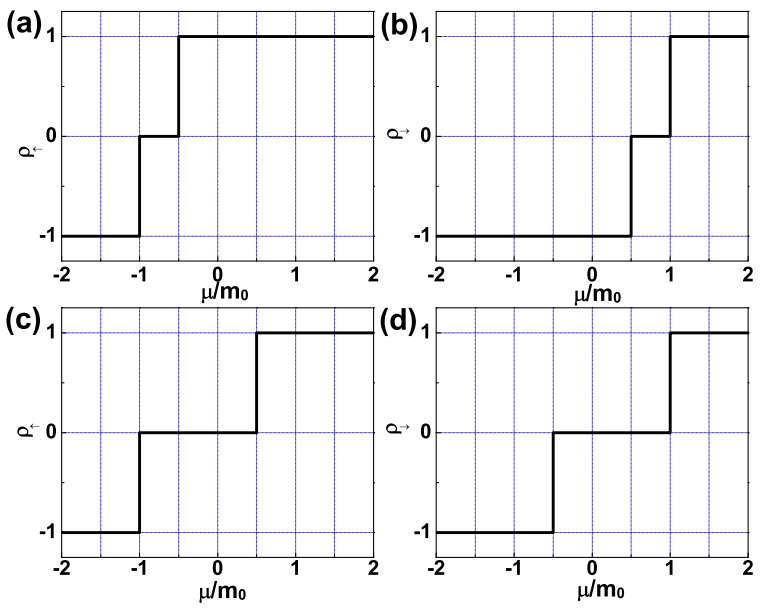

FIG. 6. (Color online) The spin-atomic density in units $\mathcal{B} / \phi_{0}$ as a function of the normalized chemical potential $\mu / m_{0}$ with the definition $\hbar v_{F}^{2}|e \mathcal{B}|=4\left|m_{0}\right|^{2}$. (a) and (b) correspond the QSH phase case with $\sqrt{3} \lambda_{S O}>3 \kappa>0$; (c) and (d) correspond the normal phase case with $\sqrt{3} \lambda_{S O}<3 \kappa<0 . \quad \rho_{\uparrow}\left(\rho_{\downarrow}\right)$ denotes the up-(down-)spin-atomic density.

From the Landau energy levels obtained at Eq. (11), we get the density [12] for every Dirac Hamiltonian (10) as

$$
\begin{aligned}
\rho_{s \alpha} & =\sum_{n=0}^{+\infty} \frac{\operatorname{sgn}(\mu) \mathcal{B}}{2 \phi_{0}}\left[\Theta\left(|\mu|-E_{n+1}\right)+\Theta\left(|\mu|-E_{n}\right)\right] \\
& +\frac{\mathcal{B}}{2 \phi_{0}} \frac{m_{s \alpha}}{\left|m_{s \alpha}\right|} \Theta\left(\left|m_{s \alpha}\right|-|\mu|\right),
\end{aligned}
$$

where $\Theta$ stands for the unit step function, $\mu$ is the chemical potential and $\phi_{0}$ the flux quantum. The second term of Eq. (23) is the atom density coming from $n=0$ (zero-mode), similarly the discuss of the Cherm number (Seeing Fig. 2). The calculated spin-atomic density $\rho_{\alpha}=\rho_{+\alpha}+\rho_{-\alpha}(\alpha=\uparrow, \downarrow)$ in unit of $\mathcal{B} / \phi_{0}$ is plotted as a function of the normalized chemical potential $\mu / m_{0}$ (for $\hbar v_{F}^{2}|e \mathcal{B}|=4\left|m_{0}\right|^{2}$ ) in Fig. 6. It is essential that the spatial density profile is uniquely determined by the function $\rho\left(\mu / m_{0}\right)$ in the local density approximation, which is typically well satisfied for trapped fermions. We focus in the point $\mu=0$ where the Dirac mass makes its central effect on the density or Chern number. When the system is in QSH phase, as an example, we take the parameters $\sqrt{3} \lambda_{\mathrm{SO}}>3 \kappa>0$, specially assuming $\left|m_{+\uparrow}\right|=\left|m_{-\downarrow}\right|=0.5\left|m_{-\uparrow}\right|=0.5\left|m_{+\downarrow}\right|=0.5 m_{0}$, then we have $m_{-\uparrow}>m_{+\uparrow}>0>m_{-\downarrow}>m_{+\downarrow}$. The spin-atomic density is shown in Fig. 6(a) and (b). It is easy to see that the up-spin-atomic density $\rho_{\uparrow}=\mathcal{B} / \phi_{0}>0$ (Fig. $6(\mathrm{a})$ ) and the down-one $\rho_{\downarrow}=-\mathcal{B} / \phi_{0}<0$ (Fig. 6(b)). For the normal phase, we take $3 \kappa>\sqrt{3} \lambda_{\mathrm{SO}}>0$ and assume $\left|m_{+\uparrow}\right|=\left|m_{-\downarrow}\right|=0.5\left|m_{-\uparrow}\right|=0.5\left|m_{+\downarrow}\right|=0.5 m_{0}$, which means $m_{-\uparrow}>m_{+\uparrow}>0>m_{-\downarrow}>m_{+\downarrow}$. We obtain $\rho_{\uparrow, \downarrow}=0$ (Fig. 6(c) and (d)). The spin-atomic density shows the similar relation comparing to the conclusion of spin Chern number theroy. This is not surprising because ac- 
cording to the Streda formula and from Eq. (23), it is easy to obtain the relation between the spin Chern number and the spin-atomic density as

$$
C_{\alpha}=\rho_{\alpha} \phi_{0} / \mathcal{B}
$$

This formula provides us the approach to measure whether the system is in the QSH phase. Firstly, we measured the spin-atomic density and denoted it as $\rho_{\uparrow, \downarrow}^{0}$ at $\mu=0$ in the absence of $\mathcal{B}$. Then the optical lattice is rotated to generate the effective uniform magnetic field $\mathcal{B}$, and the new density of the cold atoms $\rho_{\uparrow, \downarrow}^{1}$ is measured again. If $\rho_{\uparrow}^{1}>\rho_{\uparrow}^{0}$ and $\rho_{\downarrow}^{1}<\rho_{\downarrow}^{0}$, the system is in QSH insulator phase. However, if $\rho_{\uparrow, \downarrow}^{1}=\rho_{\uparrow, \downarrow}^{0}$, the system is in the normal insulator phase. Since the density difference is actually quantized in units $\mathcal{B} / \phi_{0}$, the above method could be rather robust.

It is clear from Eq. (24) that the total Chern number $C=C_{\uparrow}+C_{\downarrow}=0$ and thus the direct detection of the QSH phase is a challenge in an electronic system. However the QSH phase can be directly verified in the atomic systems since the densities $\rho_{\uparrow}$ and $\rho_{\downarrow}$ can be separately detected [5]. To experimentally detect the spin-atomic density, we need first transfer the dressed state $\left|\chi_{\downarrow}\right\rangle$ to a different hyperfine level $|F\rangle$ which is coupled to the excited state $|3\rangle$ by a laser pulse (with a Rabi frequency $\Omega_{F}$ ), as seen in Fig. 5(b). This pulse, together with the original laser beams $\Omega_{p}$ and $\Omega_{c}$, make a Raman transition with an effective Hamiltonian $H_{R}=\left(\Omega_{F}^{*} \Omega / \Delta\right)\left|\chi_{\downarrow}\right\rangle\langle F|+$ H.c. (note that the state $\left|\chi_{\uparrow}\right\rangle$ is still decoupled because of the phase relation between $\Omega_{p}$ and $\Omega_{c}$ ) [5]. Although the form of the state $\left|\chi_{\downarrow}\right\rangle$ is spatially varying, the Rabi frequency $\Omega$ (and thus also $\Omega_{F}^{*} \Omega / \Delta$ ) is spatially constant. A complete Raman transition with a $\pi$ pulse will transfer all of the atoms being in the dressed state $\left|\chi_{\downarrow}\right\rangle$ to hyperfine state $|F\rangle$. After this operation, the initial different dressed spin states are mapped to different hyperfine levels, and the populations in different atomic hyperfine levels can be separately imaged with the known experimental techniques.

\section{SUMMARY}

In summary, we have proposed a model which promises to host the transition from the QSH insulator phase to the normal insulator phase in the 2D Kagomé optical lattice. The model includes two kind of periodic perturbations, i.e., a nearest-neighbor intrinsic SO coupling and a trimerized Hamiltonian. The competition between them determines the system's phase. Then we demonstrate that the lattice SO coupling can be simulated by the laser-induced-gauge-field method and give the specific laser setting and parameters to realize the intrinsic SO coupling. Furthermore, we have established the relation between spin Chern number and spin-atomic density and then we can detect the spin Chern number through the standard density-profile technique used in atomic system.

\section{ACKNOWLEDGEMENT}

S. L. Zhu was supported in part by NSF of China (No 10974059) and the State Key Program for Basic Research of China (Nos.2006CB921801 and 2007CB925204). This work was supported by NSF of China under Grants Nos. 10874235, 10934010, 60978019, and by NKBRSFC under Grants Nos. 2009CB930701, 2010CB922904, 2011CB921500.
[1] M. Lewenstein, A. Sanpera, V. Ahufinger, B. Damski, A. Sen(De), U. Sen, Advances in Physics 56, 243 (2007).

[2] J. Ruseckas, G. Juzeliūnas, P. Öhberg, and M. Fleischhauer, Phys. Rev. Lett. 95, 010404 (2005).

[3] K. Osterloh, M. Baig, L. Santos, P. Zoller, and M. Lewenstein, Phys. Rev. Lett. 95, 010403 (2005).

[4] G. Juzeliūnas, J. Ruseckas, P. Öhberg, and M. Fleischhauer, Phys. Rev. A 73, 025602 (2006).

[5] S. L. Zhu, H. Fu, C. J. Wu, S. C. Zhang, and L. M. Duan, Phys. Rev. Lett. 97, 240401 (2006); S. L. Zhu, D. W. Zhang, and Z. D. Wang, Phys. Rev. Lett. 102, 210403 (2009).

[6] Y. J. Lin, R. L. Compton, A. R. Perry, W. D. Phillips, J.V. Porto, and I. B. Spielman, Phys. Rev. Lett. 102, 130401 (2009).

[7] Y. J. Lin, R. L. Compton, K. Jiménez-García, J. V. Porto, and I. B. Spielman, Nature 462, 628 (2009).

[8] K. v. Klitzing, G. Dorda, and M. Pepper, Phys. Rev. Lett. 45, 494 (1980); D. C. Tsui, H. L. Stormer, and A. C. Gossard, Phys. Rev. Lett. 48, 1559 (1982); D. J. Thouless, M. Kohmoto, M. P. Nightingale, and M. den Nijs, Phys. Rev. Lett. 49, 405 (1982); B. I. Halperin, Phys.
Rev. B 25, 2185 (1982); Q. Niu, D. J. Thouless, and Y. S. Wu, Phys. Rev. Lett. 31, 3372 (1985); Y. Hatsugai, Phys. Rev. Lett. 71, 3697 (1993).

[9] T. D. Stanescu, V. Galitski, J. Y. Vaishnav, C. W. Clark, and S. D. Sarma, Phys. Rev. A 79, 053639 (2009).

[10] A. Bermudez, N. Goldman, A. Kubasiak, M. Lewenstein and M. A. Martin-Delgado, New J. Phys. 12, 033041 (2010).

[11] I. I. Satija, Indubala, D. C. Dakin, J. Y. Vaishnav, and C. W. Clark, Phys. Rev. A 77, 043410 (2008).

[12] L. B. Shao, S. L. Zhu, L. Sheng, D.Y. Xing, and Z. D. Wang, Phys. Rev. Lett. 101, 246810 (2008).

[13] X. J. Liu, X. Liu, C. Wu, and J. Sinova, Phys. Rev. A 81, 033622 (2010).

[14] N. Goldman, I. Satija, P. Nikolic, A. Bermudez, M. A. Martin-Delgado, M. Lewenstein, and I. B. Spielman, arXiv: 1002.0219v2; A. Bermudez, L. Mazza, M. Rizzi, N. Goldman, M. Lewenstein, and M. A. Martin-Delgado, arXiv: 1004.5101v1.

[15] C. N. Varney, K. Sun, M. Rigol, and V. Galitski, arXiv: 1007.3502v1. 
[16] T. D. Stanescu, V. Galitski, and S. D. Sarma, arXiv:0912.3559v1.

[17] F. D. M. Haldane, Phys. Rev. Lett. 61, 2015 (1988).

[18] C. L. Kane and E. J. Mele, Phys. Rev. Lett. 95, 146802 (2005); 95, 226801 (2005).

[19] B. A. Bernevig, T. L. Hughes, and S. C. Zhang, Science 314, 1757 (2006).

[20] L. Fu, C. L. Kane, and E. J. Mele, Phys. Rev. Lett. 98, 106803 (2007).

[21] L. Fu, and C. L. Kane, Phys. Rev. B 76, 045302 (2007).

[22] J. Moore, Nature physics 5, 378 (2009); Nature 460, 1090 (2009); ibid. 464, 194 (2010).

[23] M. Büttiker, Science, 325, 278 (2009).

[24] S. Murakami, Phys. Rev. Lett. 97, 236805 (2006).

[25] M. König, S. Wiedmann, C. Breüne, A. Roth, H. Buhmann, L. W. Molenkamp, X. L. Qi, S. C. Zhang, Science 318, 766 (2007).

[26] D. Hsieh, D. Qian, L. Wray, Y. Xia, Y. S. Hor, R. J. Cava and M. Z. Hasan, Nature 452, 970 (2008); D. Hsieh, Y. Xia, D. Qian, L. Wray, J. H. Dil, F. Meier, J. Osterwalder, L. Patthey, J. G. Checkelsky, N. P. Ong, A. V. Fedorov, H. Lin, A. Bansil, D. Grauer, Y. S. Hor, R. J. Cava, and M. Z. Hasan, Nature 460, 1101 (2009); D. Hsieh, Y. Xia, L. Wray, D. Qian, A. Pal, J. H. Dil, J. Osterwalder, F. Meier, G. Bihlmayer, C. L. Kane, Y. S. Hor, R. J. Cava, M. Z. Hasan, Science 323, 919 (2009); P. Roushan, J. Seo, C. V. Parker, Y. S. Hor, D. Hsieh, D. Qian, A. Richardella, M. Z. Hasan, R. J. Cava and A. Yazdani, Nature 460, 1106 (2009).

[27] H. Zhang, C. X. Liu, X. L. Qi, X. Dai, Z. Fang, and S. C. Zhang, Nature physics 5, 438 (2009); X. L. Qi, R. Li, J. Zang, S. C. Zhang, Science 323, 1184 (2009); Y. L. Chen, J. G. Analytis, J. H. Chu, Z. K. Liu, S. K. Mo, X. L. Qi, H. J. Zhang, D. H. Lu, X. Dai, Z. Fang, S. C. Zhang, I. R. Fisher, Z. Hussain, Z.-X. Shen, Science 325,
178 (2009).

[28] A. S. Sørensen, E. Demler, and M. D. Lukin, Phys. Rev. Lett. 94, 086803 (2005).

[29] R. N. Palmer, and D. Jaksch, Phys. Rev. Lett. 96, 180407 (2006).

[30] R. O. Umucallar, H. Zhai, and M. Ö. Oktel, Phys. Rev. Lett. 100, 070402 (2008).

[31] X. J. Liu, M. F. Borunda, X. Liu, and J. Sinova, Phys. Rev. Lett. 102, 046402 (2009); X. J. Liu, X. Liu, L. C. Kwek, and C. H. Oh, Phys. Rev. Lett. 98, 026602 (2007).

[32] Y. Li, C. Bruder, and C. P. Sun, Phys. Rev. Lett. 99, 130403 (2007).

[33] T. D. Stanescu, C. Zhang, and V. Galitski, Phys. Rev. Lett. 99, 110403 (2007).

[34] H. M. Guo and M. Franz, Phys. Rev. B 80, 113102 (2009).

[35] Z. Wang and P. Zhang, Phys. Rev. B 76, 064406 (2007); ibid. 77, 125119 (2008).

[36] D. N. Sheng, Z. Y. Weng, L. Sheng, and F. D. M. Haldane, Phys. Rev. Lett. 97, 036808 (2006).

[37] A. Mielke, J. Phys. A 24, L73 (1991); 24, 3311 (1991); 25, 4335 (1992).

[38] L. Santos, M. A. Baranov, J. I. Cirac, H. U. Everts, H. Fehrmann, and M. Lewenstein, Phys. Rev. Lett. 93, 030601 (2004).

[39] B. Damski, H. Fehrmann, H. U. Everts, M. Baranov, L. Santos, and M. Lewenstein, Phys. Rev. A 72, 053612 (2005).

[40] C. Lee, T. J. Alexander, and Y. S. Kivshar, Phys. Rev. Lett. 97, 180408 (2006).

[41] N. Goldman, A. Kubasiak, A. Bermudez, P. Gaspard, M. Lewenstein, and M. A. Martin-Delgado, Phys. Rev. Lett. 103, 035301 (2009). 